\title{
Distributions of Points and Large Quadrangles (Extended Abstract)
}

\author{
Hanno Lefmann \\ Fakultät für Informatik, TU Chemnitz, D-09107 Chemnitz, Germany \\ lefmann@informatik.tu-chemnitz.de
}

\begin{abstract}
We consider a variant of Heilbronn's triangle problem by asking, given any integer $n \geq 4$, for the supremum $\Delta_{4}(n)$ of the minimum area determined by the convex hull of some four of $n$ points in the unit-square $[0,1]^{2}$ over all distributions of $n$ points in $[0,1]^{2}$. Improving the lower bound $\Delta_{4}(n)=\Omega\left(1 / n^{3 / 2}\right)$ of Schmidt [19], we will show that $\Delta_{4}(n)=\Omega\left((\log n)^{1 / 2} / n^{3 / 2}\right)$ as asked for in [5], by providing a deterministic polynomial time algorithm which finds $n$ points in the unit-square $[0,1]^{2}$ that achieve this lower bound.
\end{abstract}

\section{Introduction}

The problem of Heilbronn asks for a distribution of $n$ points in the unit-square $[0,1]^{2}$ (or unit-ball) such that the minimum area of a triangle determined by three of these $n$ points is as large as possible. Let $\Delta_{3}(n)$ denote the supremum of this minimum area of a triangle over all distributions of $n$ points in $[0,1]^{2}$. In considering for primes $n$ the points $1 / n \cdot\left(i \bmod n, i^{2} \bmod n\right), i=$ $0, \ldots, n-1$, on the moment-curve one easily sees that $\Delta_{3}(n)=\Omega\left(1 / n^{2}\right)$. While for some time this lower bound was believed to be also the upper bound for $\Delta_{3}(n)$, Komlós, Pintz and Szemerédi [11] showed by probabilistic arguments that $\Delta_{3}(n)=\Omega\left(\log n / n^{2}\right)$, see Bertram-Kretzberg, Hofmeister and this author [5] for a deterministic polynomial time algorithm achieving this lower bound $\Delta_{3}(n)=\Omega\left(\log n / n^{2}\right)$. Upper bounds on $\Delta_{3}(n)$ were given by Roth [14-18] and Schmidt [19] and, improving these earlier results, the currently best upper bound $\Delta_{3}(n)=O\left(2^{c \sqrt{\log n}} / n^{8 / 7}\right)$, where $c>0$ is a constant, is due to Komlós, Pintz and Szemerédi [10]. Recently, Jiang, Li and Vitany [9] showed by using methods from Kolmogorov complexity that if $n$ points are distributed uniformly at random and independently of each other in the unit-square $[0,1]^{2}$, then the expected value of the minimum area of a triangle formed by some three of these $n$ random points is equal to $\Theta\left(1 / n^{3}\right)$. As indicated in [9], this result might be of use to measure the affiancy of certain Monte Carlo methods for determining fair market values of derivatives.

Higher dimensional extensions of Heilbronn's triangle problem were investigated by Barequet $[2,3]$, who considered for fixed integers $d \geq 2$ the minimum volumes of simplices among $n$ points in the $d$-dimensional unit-cube $[0,1]^{d}$, maximized over all distributions of $n$ points in [0,1] $]^{d}$, see also [12], [13] and Brass [6]. 
A variant of Heilbronn's problem asks, given a fixed integer $k \geq 3$, for the supremum $\Delta_{k}(n)$ of the minimum area of the convex hull of any $k$ points in a distribution of $n$ points in the unit-square $[0,1]^{2}$, where the supremum is over all distributions of $n$ points in $[0,1]^{2}$. For $k=4$, Schmidt [19] proved the nonconstructive lower bound $\Delta_{4}(n)=\Omega\left(1 / n^{3 / 2}\right)$. In [5] a deterministic polynomial time algorithm was given, which finds, given an integer $k \geq 3$, for any integer $n \geq k$ a configuration of $n$ points in $[0,1]^{2}$ achieving the lower bound $\Delta_{k}(n)=\Omega\left(1 / n^{(k-1) /(k-2)}\right)$.

Here we provide for $k=4$ a deterministic polynomial time algorithm which achieves the lower bound $\Delta_{4}(n)=\Omega\left((\log n)^{1 / 2} / n^{3 / 2}\right)$, as asked for in [5], hence improving also the lower bound $\Delta_{4}(n)=\Omega\left(1 / n^{3 / 2}\right)$ of Schmidt [19] by a factor of $\Theta\left((\log n)^{1 / 2}\right)$.

Theorem 1. For integers $n \geq 4$ one can find deterministically in time $O\left(n^{13 / 2+\delta}\right)$ for any $\delta>0$ some $n$ points in the unit-square $[0,1]^{2}$ such that the minimum area of the convex hull determined by some four of these $n$ points is at least $\Omega\left((\log n)^{1 / 2} / n^{3 / 2}\right)$.

Although the mathematics in connection with Heilbronn-type problems seems to be hard, these problems are of interest from the algorithmic point of view as one can test the power of certain algorithmic methods with these problems, i.e. here we will use an approach by approximating the independence number of linear (or uncrowded) hypergraphs. There is some vague indication that perhaps our algorithm finds an asymptotically best possible distribution of points, but at present only $\Delta_{4}(n)=O(1 / n)$ is known.

\section{Basic Facts}

For distinct points $P, Q \in[0,1]^{2}$ let $P Q$ denote the line through $P$ and $Q$. Let dist $(P, Q)$ denote the Euclidean distance between the points $P$ and $Q$. For points $P_{1}, \ldots, P_{l} \in[0,1]^{2}$ let area $\left(P_{1}, \ldots, P_{l}\right)$ be the area of the convex hull of the points $P_{1}, \ldots, P_{l}$. A strip $S$ centered at the line $P Q$ of width $w$ is the set of all points which have distance at most $w / 2$ from the line $P Q$.

Lemma 1. Let $P_{1}, \ldots, P_{l} \in[0,1]^{2}$ be points. If area $\left(P_{1}, \ldots, P_{l}\right) \leq A$, then area $\left(P_{1}, \ldots, P_{l-1}\right) \leq A$.

Proof. This follows by monotonicity, as by definition area $\left(P_{1}, \ldots, P_{l}\right)$ is the area of the convex hull of the points $P_{1}, \ldots, P_{l}$.

Lemma 2. Let $P_{1}, \ldots, P_{l} \in[0,1]^{2}$ be distinct points no three on a line such that area $\left(P_{1}, \ldots, P_{l}\right) \leq A$. Then for any two distinct points $P_{i}$ and $P_{j}$, each other point $P_{k}, k \neq i, j$, lies in a strip centered at the line $P_{i} P_{j}$ of width 4. $A / \operatorname{dist}\left(P_{i}, P_{j}\right)$.

Proof. Otherwise, by Lemma 1 it is $A \geq$ area $\left(P_{1}, \ldots, P_{l}\right) \geq$ area $\left(P_{i}, P_{j}, P_{k}\right)>$ $1 / 2 \cdot \operatorname{dist}\left(P_{i}, P_{j}\right) \cdot(2 \cdot A) / \operatorname{dist}\left(P_{i}, P_{j}\right)=A$, a contradiction. 
Definition 1. Let $\mathcal{G}=(V, \mathcal{E})$ be a $k$-uniform hypergraph, i.e. $|E|=k$ for each edge $E \in \mathcal{E}$. An unordered pair $\left\{E, E^{\prime}\right\}$ of distinct edges $E, E^{\prime} \in \mathcal{E}$ is called a 2-cycle if $\left|E \cap E^{\prime}\right| \geq 2$. A 2-cycle $\left\{E, E^{\prime}\right\}$ in $\mathcal{G}$ is called $(2, i)$-cycle if $\left|E \cap E^{\prime}\right|=i$, $i=2, \ldots, k-1$. The hypergraph $\mathcal{G}$ is called linear if it does not contain any 2cycles. The independence number $\alpha(\mathcal{G})$ of $\mathcal{G}$ is the largest size of a subset $I \subseteq V$ which contains no edges from $\mathcal{E}$, i.e. $E \not \subset I$ for each edge $E \in \mathcal{E}$.

\section{A Deterministic Algorithm}

Here we will prove Theorem 1. By looking at the existing literature, probabilistic existence arguments using evaluations of certain integrals over $[0,1]^{2}$ might be possible to get an improvement on $\Delta_{4}(n)=\Omega\left(1 / n^{3 / 2}\right)$, however, whether this approach might be successful or not, due to the continuity of the calculations, this does not result in a deterministic algorithm. Therefore, to provide a polynomial time algorithm, which finds $n$ points in the unit-square $[0,1]^{2}$ that achieve the lower bound $\Delta_{4}(n)=\Omega\left((\log n)^{1 / 2} / n^{3 / 2}\right)$, we will discretize the search space $[0,1]^{2}$ by considering the standard $T \times T$-grid, where $T=n^{1+\alpha}$ for some constant $\alpha>0$, which will be specified later. However, with this discretization we have to take care of collinear triples or quadruples of grid-points in the $T \times T$-grid, as the area of a collinear quadruple of grid-points is equal to zero.

We will transform our problem into a problem of finding in a suitably defined hypergraph a large independent set. For some value $A>0$, which will be specified later, we form a hypergraph $\mathcal{G}=\mathcal{G}(A)=\left(V, \mathcal{E}_{3} \cup \mathcal{E}_{4}\right)$ which contains 3-element and 4-element edges. The vertex set $V$ consists of the $T^{2}$ grid-points from the $T \times T$-grid. The edge sets $\mathcal{E}_{3}$ and $\mathcal{E}_{4}$ are defined as follows: $\{P, Q, R\} \in \mathcal{E}_{3}$ if and only if the grid-points $P, Q, R \in V$ lie on a single line, i.e. are collinear, and $\{P, Q, R, S\} \in \mathcal{E}_{4}$ if and only if no three of the grid-points $P, Q, R, S \in V$ are collinear and area $(P, Q, R, S) \leq A$. In this hypergraph $\mathcal{G}=\mathcal{G}(A)$ we want to find a certain induced subhypergraph $\mathcal{G}^{* *}=\left(V^{* *}, \mathcal{E}_{4}^{* *}\right)$ of $\mathcal{G}$, which does not contain any 3-element edges anymore, hence no three distinct grid-points from the vertex set $V^{* *}$ are collinear. An independent set $I \subseteq V^{* *}$ in this induced hypergraph $\mathcal{G}^{* *}=\left(V^{* *}, \mathcal{E}_{4}^{* *}\right)$ yields $|I|$ grid-points in the $T \times T$-grid, such that the area of the convex hull of each four distinct grid-points is bigger than $A$. We are looking for a large independent set $I \subseteq V^{* *}$ in $\mathcal{G}^{* *}$.

An essential tool in our arguments is the following algorithmic version from Bertram-Kretzberg and this author [4] of a deep result of Ajtai, Komlós, Pintz, Spencer and Szemerédi [1], see also [7] and [8].

Theorem 2. Let $\mathcal{G}=(V, \mathcal{E})$ be a $k$-uniform linear hypergraph with average degree $t^{k-1}=k \cdot|\mathcal{E}| /|V|$. Then one can find for any $\delta>0$ in time $O(|V|+|\mathcal{E}|+$ $\left.|V|^{3} / t^{3-\delta}\right)$ an independent set $I \subseteq V$ with $|I|=\Omega\left(|V| / t \cdot(\log t)^{1 /(k-1)}\right)$.

The difficulty in our arguments does not lie in the invention of a new algorithm but rather to prove that the algorithm from Theorem 2 can be applied and yields a solution with the desired quality. However, our hypergraph $\mathcal{G}=\mathcal{G}(A)$ is not linear and contains many 2-cycles. The strategy will be to find a certain 
induced subhypergraph $\mathcal{G}^{*}$ of our hypergraph $\mathcal{G}=\left(V, \mathcal{E}_{3} \cup \mathcal{E}_{4}\right)$, which is linear. Therefore, to apply Theorem 2 we will carefully count in the hypergraph $\mathcal{G}=$ $\mathcal{G}(A)$ the numbers $\left|\mathcal{E}_{3}\right|$ and $\left|\mathcal{E}_{4}\right|$ of 3- and 4-element edges, respectively. Also, we will give upper bounds on the numbers of 2-cycles arising from the 4-element edges $E \in \mathcal{E}_{4}$. Then in a certain induced subhypergraph $\mathcal{G}^{*}$ of $\mathcal{G}$ we will destroy in one step all induced 3 -element edges and all 2-cycles. The resulting induced subhypergraph $\mathcal{G}^{* *}$ of $\mathcal{G}$ has not too few vertices and does not contain any 2cycles anymore and at this point we can apply the algorithm from Theorem 2 to $\mathcal{G}^{* *}$.

For positive integers $h$ and $s$ let gcd $(h, s) \geq 0$ denote the greatest common divisor of $h$ and $s$. For a grid-point $P$ in the $T \times T$-grid let $p_{x}$ and $p_{y}$ denote its $x$ - and $y$-coordinate, respectively. We define a lexicographic order $\leq_{l e x}$ on the grid-points of the $T \times T$-grid: for grid-points $P=\left(p_{x}, p_{y}\right)$ and $Q=\left(q_{x}, q_{y}\right)$ let

$$
P \leq_{l e x} Q \Longleftrightarrow\left(p_{x}<q_{x}\right) \text { or }\left(p_{x}=q_{x} \text { and } p_{y}<q_{y}\right) .
$$

Notice that for grid-points $P=\left(p_{x}, p_{y}\right)$ and $Q=\left(q_{x}, q_{y}\right)$ with $P \leq_{l e x} Q$ there are exactly (gcd $\left.\left(q_{x}-p_{x}, q_{y}-p_{y}\right)-1\right)$ grid-points on the segment $[P, Q]$ excluding the endpoints $P$ and $Q$.

We will use the following result from [5].

Lemma 3. For two grid-points $P=\left(p_{x}, p_{y}\right)$ and $R=\left(r_{x}, r_{y}\right)$ with $P \leq_{l e x} R$ in the $T \times T$-grid, where $s:=r_{x}-p_{x} \geq 0$, the following hold:

(a) There are at most $4 \cdot A$ grid-points $Q$ in the $T \times T$-grid such that

(i) $P \leq_{\text {lex }} Q \leq_{\text {lex }} R$, and

(ii) $P, Q, R$ are not collinear, and

(iii) area $(P, Q, R) \leq A$.

(b) The number of grid-points $Q$ in the $T \times T$-grid which fulfill only conditions (ii) and (iii) from (a) is at most $12 \cdot A \cdot T / s$ for $s>0$ and at most $4 \cdot A \cdot T$ for $s=0$.

First we will estimate the numbers $\left|\mathcal{E}_{3}\right|$ and $\left|\mathcal{E}_{4}\right|$ of 3- and 4-element edges, respectively, in the hypergraph $\mathcal{G}=\left(V, \mathcal{E}_{3} \cup \mathcal{E}_{4}\right)$.

Lemma 4. The number $\left|\mathcal{E}_{3}\right|$ of 3-element edges in the hypergraph $\mathcal{G}=\left(V, \mathcal{E}_{3} \cup\right.$ $\left.\mathcal{E}_{4}\right)$ satisfies

$$
\left|\mathcal{E}_{3}\right| \leq c_{3} \cdot T^{4} \cdot \log T .
$$

We remark that in [5] an upper bound of $O\left(T^{4+\varepsilon}\right)$, for any $\varepsilon>0$, on the number of collinear triples of grid-points in the $T \times T$-grid was proved.

Proof. Let $P, Q, R$ be grid-points from the $T \times T$-grid, where $P \leq_{\text {lex }} Q \leq_{l e x} R$. Set $s=: r_{x}-p_{x} \geq 0$ and $h:=r_{y}-p_{y}$. We have $\{P, Q, R\} \in \mathcal{E}_{3}$ if and only if $P, Q, R$ are collinear. For $h=0$ or $s=0$ the number of collinear triples $P, Q, R$ of grid-points is at most $O\left(T^{4}\right)$, as we can choose one of the $2 \cdot T$ horizontal or vertical lines and on each of these at most $O\left(T^{3}\right)$ triples of grid-points. 
Let $h, s \neq 0$. A grid-point $P$ can be chosen in at most $T^{2}$ ways. Given the gridpoint $P$, any pair $(s, h)$ of integers with $1 \leq h \leq s \leq T$ determines a grid-point $R$. Those pairs $(s, h)$ of integers with $0 \leq-h \leq s \leq T$ or $1 \leq s<|h| \leq T$ will be taken into account by an additional constant factor using rotation symmetry. On the segment $[P, R]$ there are at most gcd $(h, s)$ grid-points $Q$ exluding $P$ and $R$. Thus, for a constant $c^{\prime}>0$ the number of collinear triples of grid-points in the $T \times T$-grid is at most

$$
c^{\prime} \cdot T^{2} \cdot \sum_{s=1}^{T} \sum_{h=1}^{s} \operatorname{gcd}(h, s) .
$$

Each divisor $d$ of $s$ divides at most $s / d$ positive integers $x$ with $x \leq s$, namely the integers $i \cdot d, i=1, \ldots,\lfloor s / d\rfloor$, hence we infer for a constant $c_{3}>0$ :

$$
\begin{aligned}
& c^{\prime} \cdot T^{2} \cdot \sum_{s=1}^{T} \sum_{h=1}^{s} \operatorname{gcd}(h, s) \leq c^{\prime} \cdot T^{2} \cdot \sum_{s=1}^{T} \sum_{d \mid s} \frac{s}{d} \cdot d \leq c^{\prime} \cdot T^{2} \cdot \sum_{s=1}^{T} s \sum_{d \mid s} 1 \leq \\
\leq & c^{\prime} \cdot T^{2} \cdot \sum_{d=1}^{T} \sum_{i=1}^{\lfloor T / d\rfloor} i \cdot d \leq c^{\prime} \cdot T^{2} \cdot \sum_{d=1}^{T} d \cdot \frac{T^{2}}{d^{2}}=c^{\prime} \cdot T^{4} \cdot \sum_{d=1}^{T} \frac{1}{d} \leq \\
\leq & c_{3} \cdot T^{4} \cdot \log T .
\end{aligned}
$$

Lemma 5. The number $\left|\mathcal{E}_{4}\right|$ of unordered quadruples $P_{1}, P_{2}, P_{3}, P_{4}$ of distinct grid-points in the $T \times T$-grid with area $(P, Q, R, S) \leq A$, where no three of the grid-points $P_{1}, P_{2}, P_{3}, P_{4}$ are collinear, fulfills

$$
\left|\mathcal{E}_{4}\right| \leq c_{4} \cdot A^{2} \cdot T^{4}
$$

Proof. We can assume that $P_{1} \leq_{\text {lex }} P_{3} \leq_{\text {lex }} P_{4} \leq_{\text {lex }} P_{2}$. Let $s:=p_{2, x}-p_{1, x} \geq 0$ and $h:=p_{2, y}-p_{1, y}$. If $s=0$, then the grid-points $P_{1}, P_{2}, P_{3}, P_{4}$ are collinear, hence we have $s \neq 0$. By rotation symmetry, which we take into account by an additional constant factor, we can assume that $0 \leq h \leq s \leq T$.

If area $\left(P_{1}, P_{2}, P_{3}, P_{4}\right) \leq A$, then by Lemma 2 we have area $\left(P_{1}, P_{2}, P_{3}\right) \leq A$ and area $\left(P_{1}, P_{2}, P_{4}\right) \leq A$. There are $T^{2}$ choices for the grid-point $P_{1}$. Given the grid-point $P_{1}$, any pair $(s, h) \neq(0,0)$ of integers with $0 \leq h \leq s \leq T$ determines a grid-point $P_{2}$. Since $P_{1} \leq_{\text {lex }} P_{3} \leq_{\text {lex }} P_{4} \leq_{\text {lex }} P_{2}$ and since no three of the gridpoints $P_{1}, P_{2}, P_{3}, P_{4}$ are collinear, by Lemma 3 (a) there are at most $4 \cdot A$ choices for the grid-points $P_{3}$ and $P_{4}$ each. Thus, we obtain for constants $c^{\prime}, c_{4}>0$ for the number $\left|\mathcal{E}_{4}\right|$ of 4 -element edges in the hypergraph $\mathcal{G}$ an upper bound of

$\left|\mathcal{E}_{4}\right| \leq c^{\prime} \cdot T^{2} \cdot \sum_{s=1}^{T} \sum_{h=0}^{s}(4 \cdot A)^{2} \leq 16 \cdot c^{\prime} \cdot A^{2} \cdot T^{2} \cdot \sum_{s=1}^{T} \sum_{h=0}^{s} 1 \leq c_{4} \cdot A^{2} \cdot T^{4}$.

By (2) the average degree $t^{3}$ of the hypergraph $\mathcal{G}=\left(V, \mathcal{E}_{3} \cup \mathcal{E}_{4}\right)$ for the 4 -element edges $E \in \mathcal{E}_{4}$ satisfies

$$
t^{3}=\frac{4 \cdot\left|\mathcal{E}_{4}\right|}{|V|} \leq \frac{4 \cdot c_{4} \cdot A^{2} \cdot T^{4}}{T^{2}}=4 \cdot c_{4} \cdot A^{2} \cdot T^{2}:=t_{0}^{3} .
$$




\section{$3.1 \quad(2,2)-$ and $(2,3)$-Cycles}

Let $s_{2, i}\left(\mathcal{G} ; \mathcal{E}_{4}\right)$ denote the number of $(2, i)$-cycles, $i=2,3$, formed by unordered pairs of 4 -element edges $E \in \mathcal{E}_{4}$ in the hypergraph $\mathcal{G}=\left(V, \mathcal{E}_{3} \cup \mathcal{E}_{4}\right)$. Here we will give upper bounds on the numbers of these $(2,2)$ - and $(2,3)$-cycles.

Lemma 6. The number $s_{2,3}\left(\mathcal{G} ; \mathcal{E}_{4}\right)$ of $(2,3)$-cycles in the hypergraph $\mathcal{G}=\left(V, \mathcal{E}_{3} \cup\right.$ $\left.\mathcal{E}_{4}\right)$, which arise from the 4 -element edges $E \in \mathcal{E}_{4}$, satisfies

$$
s_{2,3}\left(\mathcal{G} ; \mathcal{E}_{4}\right)=O\left(A^{3} \cdot T^{4} \cdot \log T\right) .
$$

Proof. Assume that the quadruples $P_{1}, P_{2}, P_{3}, P_{4}$ and $P_{1}, P_{2}, P_{3}, P_{5}$ of grid-points determine a $(2,3)$-cycle in the hypergraph $\mathcal{G}$, where no three of these five gridpoints are collinear. Then area $\left(P_{1}, P_{2}, P_{3}, P_{4}\right) \leq A$ and area $\left(P_{1}, P_{2}, P_{3}, P_{5}\right) \leq$ $A$. We can assume that $P_{1} \leq_{\text {lex }} P_{3} \leq_{\text {lex }} P_{2}$. There are $T^{2}$ possibilities for the grid-point $P_{1}$. Given the grid-point $P_{1}$, a pair $(s, h) \neq(0,0)$ of integers, where by rotation symmetry w.l.o.g. $0 \leq h \leq s \leq T$, determines a grid-point $P_{2}$. Clearly, we have $s>0$, as for $s=0$ the grid-points $P_{1}, P_{2}, P_{3}$ are collinear. Since area $\left(P_{1}, P_{2}, P_{3}\right) \leq A$ by Lemma 2 , and since $P_{1}, P_{2}, P_{3}$ are not collinear, by Lemma 3(a) there are at most $4 \cdot A$ choices for the grid-point $P_{3}$.

With area $\left(P_{1}, P_{2}, P_{4}\right) \leq A$ and area $\left(P_{1}, P_{2}, P_{5}\right) \leq A$, and since $P_{1}, P_{2}, P_{5}$ are not collinear, by Lemma $3(\mathrm{~b})$ there are at most $12 \cdot A \cdot T / s$ choices for the gridpoints $P_{4}$ and $P_{5}$ each, hence for some constants $c^{\prime}, c^{\prime \prime}, c_{2,3}>0$ we have the following upper bound on the number $s_{2,3}\left(\mathcal{G} ; \mathcal{E}_{4}\right)$ of $(2,3)$-cycles in $\mathcal{G}$ :

$$
\begin{aligned}
& s_{2,3}\left(\mathcal{G} ; \mathcal{E}_{4}\right) \leq c^{\prime} \cdot T^{2} \cdot \sum_{s=1}^{T} \sum_{h=0}^{s}(4 \cdot A) \cdot\left(\frac{12 \cdot A \cdot T}{s}\right)^{2} \leq \\
\leq & c^{\prime \prime} \cdot A^{3} \cdot T^{4} \cdot \sum_{s=1}^{T} \sum_{h=0}^{s} \frac{1}{s^{2}} \leq c^{\prime \prime} \cdot A^{3} \cdot T^{4} \cdot \sum_{s=1}^{T} \frac{s+1}{s^{2}} \leq \\
\leq & c_{2,3} \cdot A^{3} \cdot T^{4} \cdot \log T .
\end{aligned}
$$

Next we will estimate the number of $(2,2)$-cycles in the hypergraph $\mathcal{G}$.

Lemma 7. The number $s_{2,2}\left(\mathcal{G} ; \mathcal{E}_{4}\right)$ of $(2,2)$-cycles in the hypergraph $\mathcal{G}=\left(V, \mathcal{E}_{3} \cup\right.$ $\left.\mathcal{E}_{4}\right)$, which arise from the 4 -element edges $E \in \mathcal{E}_{4}$, fulfills

$$
s_{2,2}\left(\mathcal{G} ; \mathcal{E}_{4}\right)=O\left(A^{4} \cdot T^{9 / 2}\right) .
$$

Proof. Let us denote the grid-points of two 4-element edges $E, E^{\prime} \in \mathcal{E}_{4}$, which yield a $(2,2)$-cycle in the hypergraph $\mathcal{G}$, by $P_{1}, P_{2}, P_{3}, P_{4}$ and $P_{1}, P_{2}, P_{5}, P_{6}$, where $P_{1} \leq_{\text {lex }} P_{2}$ and no three of the six points are collinear. Let $u:=\left\lceil T^{\gamma}\right\rceil$ where $0<\gamma<1$ is a constant, which will be specified later.

There are $T^{2}$ choices for the grid-point $P_{1}$. Given the grid-point $P_{1}$, any pair $(s, h) \neq(0,0)$ of integers fixes a grid-point $P_{2}$. By rotation symmetry we can assume that $s>0$ and $0 \leq h \leq s \leq T$. By Lemma 2 all grid-points $P_{3}, P_{4}, P_{5}, P_{6}$ must lie in a strip $S$ centered at the line $P_{1} P_{2}$ of width $4 \cdot A / \sqrt{h^{2}+s^{2}}$ and 
by Lemma $3(\mathrm{~b})$ this strip $S$ contains at most $12 \cdot A \cdot T / s$ grid-points $P$ from the $T \times T$-grid, such that $P_{1}, P_{2}, P$ are not collinear, hence there are at most $12 \cdot A \cdot T / s$ choices for the grid-points $P_{3}, P_{4}, P_{5}, P_{6}$ each, but we have to count more carefully.

Consider a parallelogram $\mathcal{P}_{0}=\left\{\left(p_{x}, p_{y}\right) \in S \mid p_{1, x}-u \leq_{\text {lex }} p_{x} \leq_{\text {lex }} p_{2, x}+u\right\}$ with two of its boundaries being the boundaries of the strip $S$ and with center being the middle of the segment $\left[P_{1}, P_{2}\right]$. By Lemma $3(\mathrm{a})$ this parallelogram $\mathcal{P}_{0}$ contains at most $4 \cdot A \cdot(s+2 \cdot u) / s$ grid-points $P$, such that $P_{1}, P_{2}, P$ are not collinear.

In the following we will distinguish whether some of the grid-points $P_{3}, P_{4}, P_{5}, P_{6}$ lie in the parallelogram $\mathcal{P}_{0}$ or not.

Case A: All grid-points $P_{3}, P_{4}, P_{5}, P_{6}$ satisfy $P_{3}, P_{4}, P_{5}, P_{6} \in \mathcal{P}_{0}$.

Given the grid-points $P_{1}$ and $P_{2}$, there are at most $4 \cdot A \cdot(s+2 \cdot u) / s$ choices for each of the grid-points $P_{3}, P_{4}, P_{5}, P_{6} \in \mathcal{P}_{0}$. Using $u=\left\lceil T^{\gamma}\right\rceil$, for some constants $c, c^{\prime}, c^{\prime \prime}, c^{\prime \prime \prime}, c^{\prime \prime \prime \prime}>0$ we obtain for the number of these $(2,2)$-cycles arising from $\mathcal{E}_{4}$ the following upper bound

$$
\begin{aligned}
& c \cdot T^{2} \cdot \sum_{s=1}^{T} \sum_{h=0}^{s}\left(\frac{4 \cdot A \cdot(s+2 \cdot u)}{s}\right)^{4} \\
\leq & c^{\prime} \cdot A^{4} \cdot T^{2} \cdot \sum_{s=1}^{T} \sum_{h=0}^{s}\left(1+\frac{2 \cdot u}{s}\right)^{4} \\
\leq & c^{\prime} \cdot A^{4} \cdot T^{2} \cdot \sum_{s=1}^{T} \sum_{h=0}^{s}\left(1+\frac{8 \cdot u}{s}+\frac{24 \cdot u^{2}}{s^{2}}+\frac{32 \cdot u^{3}}{s^{3}}+\frac{16 \cdot u^{4}}{s^{4}}\right) \\
\leq & c^{\prime \prime} \cdot A^{4} \cdot T^{2} \cdot \sum_{s=1}^{T}\left(s+8 \cdot T^{\gamma}+\frac{24 \cdot T^{2 \gamma}}{s}+\frac{32 \cdot T^{3 \gamma}}{s^{2}}+\frac{16 \cdot T^{4 \gamma}}{s^{3}}\right) \\
\leq & c^{\prime \prime \prime} \cdot A^{4} \cdot T^{2} \cdot\left(T^{2}+T^{1+\gamma}+T^{2 \gamma} \cdot \log T+T^{3 \gamma}+T^{4 \gamma}\right) \\
\leq & c^{\prime \prime \prime \prime} \cdot A^{4} \cdot\left(T^{4}+T^{2+4 \gamma}\right)
\end{aligned}
$$

Case B: The grid-points $P_{3}, P_{4}, P_{5}$ satisfy $P_{3}, P_{4} \in \mathcal{P}_{0}$ and $P_{5} \notin \mathcal{P}_{0}$.

Given the grid-points $P_{1}$ and $P_{2}$, there are at most $4 \cdot A \cdot(s+2 \cdot u) / s$ choices for each of the grid-points $P_{3} \in \mathcal{P}_{0}$ and $P_{4} \in \mathcal{P}_{0}$. Considering now the quadruple $P_{1}, P_{2}, P_{5}, P_{6}$ of grid-points, by Lemma $3(\mathrm{~b})$ there are at most $12 \cdot A$. $T / s$ choices for the grid-point $P_{5}=\left(p_{5, x}, p_{5, y}\right) \notin \mathcal{P}_{0}$, since $P_{1}, P_{2}, P_{5}$ are not collinear and area $\left(P_{1}, P_{2}, P_{5}\right) \leq A$. However, since now $\left|p_{1, x}-p_{5, x}\right| \geq u$ and area $\left(P_{1}, P_{2}, P_{6}\right) \leq A$, by Lemma $3(\mathrm{~b})$ the number of choices for the grid-point $P_{6}$ in the $T \times T$-grid, such that $P_{1}, P_{2}, P_{6}$ are not collinear, is at most $12 \cdot A \cdot T / u$. With $u=\left\lceil T^{\gamma}\right\rceil$ we obtain for constants $c^{\prime}, c^{\prime \prime}, c^{\prime \prime \prime}>0$ the following upper bound on the number of these $(2,2)$-cycles arising from $\mathcal{E}_{4}$ : 


$$
\begin{aligned}
& c \cdot T^{2} \cdot \sum_{s=1}^{T} \sum_{h=0}^{s} \frac{12 \cdot A \cdot T}{u} \cdot \frac{12 \cdot A \cdot T}{s} \cdot\left(\frac{4 \cdot A \cdot(s+2 \cdot u)}{s}\right)^{2} \\
\leq & c^{\prime} \cdot A^{4} \cdot T^{4} \cdot \sum_{s=1}^{T} \sum_{h=0}^{s}\left(\frac{1}{s \cdot u}+\frac{4}{s^{2}}+\frac{4 \cdot u}{s^{3}}\right) \\
\leq & c^{\prime \prime} \cdot A^{4} \cdot T^{4} \cdot \sum_{s=1}^{T}\left(\frac{1}{T \gamma}+\frac{4}{s}+\frac{4 \cdot T^{\gamma}}{s^{2}}\right) \\
\leq & c^{\prime \prime \prime} \cdot A^{4} \cdot\left(T^{5-\gamma}+T^{4+\gamma}\right) \quad \text { as } 0<\gamma<1 \text { is a constant. }
\end{aligned}
$$

Case $C$ : The grid-points $P_{3}, P_{5}$ satisfy $P_{3}, P_{5} \notin \mathcal{P}_{0}$.

Given the grid-points $P_{1}$ and $P_{2}$, we partition the strip $S$ within the $T \times T$-grid into parallelograms $\mathcal{P}_{0}, \mathcal{P}_{i}^{+}, \mathcal{P}_{i}^{-}, i=1, \ldots, l \leq T^{1-\gamma}$, which are all translates of $\mathcal{P}_{0}$, and arranged according to the order $\mathcal{P}_{l}^{+}, \mathcal{P}_{l-1}^{+}, \ldots, \mathcal{P}_{1}^{+}, \mathcal{P}_{0}, \mathcal{P}_{1}^{-}, \ldots, \mathcal{P}_{l-1}^{-}, \mathcal{P}_{l}^{-}$. Each grid-point $P=\left(p_{x}, p_{y}\right) \in \mathcal{P}_{i}^{+} \cup \mathcal{P}_{i}^{-}, i \geq 1$, satisfies $\left|p_{x}-p_{1, x}\right| \geq s+u+$ $(i-1) \cdot(s+2 \cdot u) \geq i \cdot(s+u)$ or $\left|p_{x}-p_{2, x}\right| \geq i \cdot(s+u)$. Using Lemma 3(a), each parallelogram $\mathcal{P}_{i}^{+}$or $\mathcal{P}_{i}^{-}$contains at most $4 \cdot A \cdot(s+2 \cdot u) / s$ grid-points $P$, such that $P_{1}, P_{2}, P$ are not collinear. Each grid-point $P_{3}, P_{5}$ lies in some paralelogram $\mathcal{P}_{i}^{+}$or $\mathcal{P}_{i}^{-}$for some $i \geq 1$. If $P_{3} \in \mathcal{P}_{i}^{+} \cup \mathcal{P}_{i}^{-}, i \geq 1$, then by Lemma 3(b) there are at most $12 \cdot A \cdot T /(i \cdot(s+u))$ choices for the grid-point $P_{4}$. Similarly, if $P_{5} \in \mathcal{P}_{j}^{+} \cup \mathcal{P}_{j}^{-}, j \geq 1$, there are at most $12 \cdot A \cdot T /(j \cdot(s+u))$ choices for the grid-point $P_{6}$, and we obtain for constants $c, c^{\prime}, c^{\prime \prime}, c^{\prime \prime \prime}>0$ for the number of these $(2,2)$-cycles among $\mathcal{E}_{4}$ the upper bound

$$
\begin{aligned}
& c \cdot T^{2} \cdot \sum_{s=1}^{T} \sum_{h=0}^{s} \sum_{i=1}^{l} \sum_{j=1}^{l}\left(\frac{4 \cdot A \cdot(s+2 \cdot u)}{s}\right)^{2} \cdot\left(\frac{(12 \cdot A \cdot T)^{2}}{i \cdot j \cdot(s+u)^{2}}\right) \\
\leq & c^{\prime} \cdot A^{4} \cdot T^{4} \cdot \sum_{s=1}^{T} \sum_{h=0}^{s}\left(\frac{s+2 \cdot u}{s}\right)^{2} \cdot\left(\frac{1}{s+u}\right)^{2} \cdot \sum_{i=1}^{l} \frac{1}{i} \sum_{j=1}^{l} \frac{1}{j} \\
\leq & c^{\prime \prime} \cdot A^{4} \cdot T^{4} \cdot(\log T)^{2} \cdot \sum_{s=1}^{T} \sum_{h=0}^{s}\left(\frac{1}{s+u}+\frac{2 \cdot u}{s \cdot(s+u)}\right)^{2} \\
\leq & c^{\prime \prime} \cdot A^{4} \cdot T^{4} \cdot(\log T)^{2} \cdot \sum_{s=1}^{T} \sum_{h=0}^{s} \frac{4}{s^{2}} \\
\leq & c^{\prime \prime \prime} \cdot A^{4} \cdot T^{4} \cdot(\log T)^{3},
\end{aligned}
$$

where we used $1 /(s+u)+2 \cdot u /(s \cdot(s+u)) \leq 2 / s$ for $s, u \geq 0$.

For $\gamma:=1 / 2$ the estimates $(6),(7),(8)$ yield $s_{2,2}\left(\mathcal{G} ; \mathcal{E}_{4}\right)=O\left(A^{4} \cdot T^{9 / 2}\right)$.

\subsection{Selecting a Subhypergraph}

For a suitable constant $c>0$ we set

$$
A:=\frac{c \cdot T^{2} \cdot(\log n)^{1 / 2}}{n^{3 / 2}}>1 .
$$


For a moment we will use a probabilistic argument to simplify the presentation. However, this argument will be made constructive shortly. With probability $p:=$ $T^{\varepsilon} / t_{0} \leq 1$ for a small constant $\varepsilon>0$ we pick uniformly at random and independently of each other vertices from the set $V$. Let $V^{*} \subseteq V$ be the resulting random subset of $V$ of the picked vertices and let $\mathcal{G}^{*}=\left(V^{*}, \mathcal{E}_{3}^{*} \cup \mathcal{E}_{4}^{*}\right)$ with $\mathcal{E}_{3}^{*}:=\mathcal{E}_{3} \cap\left[V^{*}\right]^{3}$ and $\mathcal{E}_{4}^{*}:=\mathcal{E}_{4} \cap\left[V^{*}\right]^{4}$ be the resulting random induced subhypergraph of $\mathcal{G}$. Let $E\left[\left|V^{*}\right|\right], E\left[\left|\mathcal{E}_{3}^{*}\right|\right], E\left[\left|\mathcal{E}_{4}^{*}\right|\right], E\left[s_{2,2}\left(\mathcal{G}^{*} ; \mathcal{E}_{4}^{*}\right)\right], E\left[s_{2,3}\left(\mathcal{G}^{*} ; \mathcal{E}_{4}^{*}\right)\right]$ denote the expected number of vertices, 3 -element edges, 4-element edges, $(2,2)$ - and $(2,3)$-cycles arising from $\mathcal{E}_{4}^{*}$, respectively, in the random subhypergraph $\mathcal{G}^{*}=\left(V^{*}, \mathcal{E}_{3}^{*} \cup \mathcal{E}_{4}^{*}\right)$ of $\mathcal{G}$. By (1), (2), (5) and (4) we infer for constants $c_{1}^{\prime}, c_{3}^{\prime}, c_{4}^{\prime}, c_{2,2}^{\prime}, c_{2,3}^{\prime}>0$ :

$$
\begin{aligned}
E\left[\left|V^{*}\right|\right] & =p \cdot T^{2}=c_{1}^{\prime} \cdot T^{4 / 3+\varepsilon} / A^{2 / 3} \\
E\left[\left|\mathcal{E}_{3}^{*}\right|\right] & =p^{3} \cdot\left|\mathcal{E}_{3}\right| \leq c_{3}^{\prime} \cdot\left(T^{4} \cdot \log T\right) \cdot T^{3 \varepsilon} /(A \cdot T)^{2} \leq \\
& \leq c_{3}^{\prime} \cdot T^{2+3 \varepsilon} \cdot \log T / A^{2} \\
E\left[\left|\mathcal{E}_{4}^{*}\right|\right] & =p^{4} \cdot\left|\mathcal{E}_{4}\right| \leq c_{4}^{\prime} \cdot\left(A^{2} \cdot T^{4}\right) \cdot T^{4 \varepsilon} /(A \cdot T)^{8 / 3} \leq \\
& \leq c_{4}^{\prime} \cdot T^{4 / 3+4 \varepsilon} / A^{2 / 3} \\
E\left[s_{2,2}\left(\mathcal{G}^{*} ; \mathcal{E}_{4}^{*}\right)\right] & \leq p^{6} \cdot s_{2,2}\left(\mathcal{G} ; \mathcal{E}_{4}\right) \leq c_{2,2}^{\prime} \cdot\left(A^{4} \cdot T^{9 / 2}\right) \cdot T^{6 \varepsilon} /(A \cdot T)^{4} \leq \\
& \leq c_{2,2}^{\prime} \cdot T^{1 / 2+6 \varepsilon} \\
E\left[s_{2,3}\left(\mathcal{G}^{*} ; \mathcal{E}_{4}^{*}\right)\right] & \leq p^{5} \cdot s_{2,3}\left(\mathcal{G} ; \mathcal{E}_{4}\right) \leq c_{2,3}^{\prime} \cdot\left(A^{3} \cdot T^{4} \cdot \log T\right) \cdot T^{5 \varepsilon} /(A \cdot T)^{10 / 3} \leq \\
& \leq c_{2,3}^{\prime} \cdot T^{2 / 3+5 \varepsilon} \cdot \log T / A^{1 / 3} .
\end{aligned}
$$

With (10), (11), (12), (13), (14) and Chernoff's and Markov's inequality there exists a subhypergraph $\mathcal{G}^{*}=\left(V^{*}, \mathcal{E}_{3}^{*} \cup \mathcal{E}_{4}^{*}\right)$ of $\mathcal{G}$ such that

$$
\begin{aligned}
\left|V^{*}\right| & \geq c_{1}^{\prime} / 2 \cdot T^{4 / 3+\varepsilon} / A^{2 / 3} \\
\left|\mathcal{E}_{3}^{*}\right| & \leq 5 \cdot c_{3}^{\prime} \cdot T^{2+3 \varepsilon} \cdot \log T / A^{2} \\
\left|\mathcal{E}_{4}^{*}\right| & \leq 5 \cdot c_{4}^{\prime} \cdot T^{4 / 3+4 \varepsilon} / A^{2 / 3} \\
s_{2,2}\left(\mathcal{G}^{*} ; \mathcal{E}_{4}^{*}\right) & \leq 5 \cdot c_{2,2}^{\prime} \cdot T^{1 / 2+6 \varepsilon} \\
s_{2,3}\left(\mathcal{G}^{*} ; \mathcal{E}_{4}^{*}\right) & \leq 5 \cdot c_{2,3}^{\prime} \cdot T^{2 / 3+5 \varepsilon} \cdot \log T / A^{1 / 3} .
\end{aligned}
$$

This probabilistic argument can be derandomized by using the method of conditional probabilities as follows. For $j=2,3$, let $\mathcal{C}_{j}$ be the (multi-)set of all $\left(8-j\right.$ )-element subsets $E \cup E^{\prime}$ of $V$ such that the pair $\left\{E, E^{\prime}\right\}$ of distinct 4element edges $E, E^{\prime} \in \mathcal{E}_{4}$ yields a $(2, j)$-cycle in $\mathcal{G}$. We enumerate the vertices of the $T \times T$-grid by $P_{1}, \ldots, P_{T^{2}}$. For each vertex $P_{i}$ we associate a parameter 
$p_{i} \in[0,1]$, and we define a potential function $F$ by

$$
\begin{aligned}
& F\left(p_{1}, \ldots, p_{T^{2}}\right):=2^{p \cdot T^{2} / 2} \cdot \prod_{i=1}^{T^{2}}\left(1-\frac{p_{i}}{2}\right)+\frac{\sum_{\{i, j, k\} \in \mathcal{E}_{3}} p_{i} \cdot p_{j} \cdot p_{k}}{5 \cdot c_{3}^{\prime} \cdot T^{2+3 \varepsilon} \cdot \log T / A^{2}}+ \\
& +\frac{\sum_{\{i, j, k, l\} \in \mathcal{E}_{4}} p_{i} \cdot p_{j} \cdot p_{k} \cdot p_{l}}{5 \cdot c_{4}^{\prime} \cdot T^{4 / 3+4 \varepsilon} / A^{2 / 3}}+\frac{\sum_{\{i, j, k, l, m, o\} \in \mathcal{C}_{2}} p_{i} \cdot p_{j} \cdot p_{k} \cdot p_{l} \cdot p_{m} \cdot p_{o}}{5 \cdot c_{2,2}^{\prime} \cdot T^{1 / 2+6 \varepsilon} / A^{2 / 3}}+ \\
+ & \frac{\sum_{\{i, j, k, l, m\} \in \mathcal{C}_{3}} p_{i} \cdot p_{j} \cdot p_{k} \cdot p_{l} \cdot p_{m}}{5 \cdot c_{2,3}^{\prime} \cdot T^{2 / 3+5 \varepsilon} \cdot \log T / A^{1 / 3}} .
\end{aligned}
$$

With the initialisation $p_{1}:=\cdots:=p_{T^{2}}:=p:=T^{\varepsilon} / t_{0}$, we infer $F(p, \ldots, p)<$ $(2 / e)^{p T^{2} / 2}+4 / 5<1$ for $p \cdot T^{2} \geq 11$. Then, using the linearity of $F\left(p_{1}, \ldots, p_{T^{2}}\right)$ in each $p_{i}$, we minimize $F\left(p_{1}, \ldots, p_{T^{2}}\right)$ by choosing one after the other $p_{i}:=0$ or $p_{i}:=1$ for $i=1, \ldots, T^{2}$, and finally we obtain $F\left(p_{1}, \ldots, p_{T^{2}}\right)<1$. Then the vertex set $V^{*}=\left\{i \in V \mid p_{i}=1\right\}$ yields an induced subhypergraph $\mathcal{G}^{*}=$ $\left(V^{*}, \mathcal{E}_{3}^{*} \cup \mathcal{E}_{4}^{*}\right)$ of $\mathcal{G}$ with $\mathcal{E}_{3}^{*}=\mathcal{E}_{3} \cup\left[V^{*}\right]^{3}$ and $\mathcal{E}_{4}^{*}=\mathcal{E}_{4} \cup\left[V^{*}\right]^{4}$, which satisfies (15), (16), (17), (18), (19), compare [4]. With (1), (2), (4), (5), (9) and using that $A>1$, the running time of this derandomization is given by

$$
O\left(|V|+\left|\mathcal{E}_{3}\right|+\left|\mathcal{E}_{4}\right|+\left|\mathcal{C}_{2}\right|+\left|\mathcal{C}_{3}\right|\right)=O\left(A^{4} \cdot T^{\frac{9}{2}}\right)=O\left(T^{\frac{25}{2}} \cdot(\log n)^{2} / n^{6}\right) .
$$

We will show next that the numbers $\left|\mathcal{E}_{3}^{*}\right|, s_{2,2}\left(\mathcal{G}^{*} ; \mathcal{E}_{4}^{*}\right)$ and $s_{2,3}\left(\mathcal{G}^{*} ; \mathcal{E}_{4}^{*}\right)$ of 3 element edges, $(2,2)$ - and $(2,3)$-cycles arising from $\mathcal{E}_{4}^{*}$ in $\mathcal{G}^{*}$, respectively, are very small compared to the number $\left|V^{*}\right|$ of vertices in $\mathcal{G}^{*}$.

Lemma 8. For $0<\varepsilon<\alpha /(1+\alpha)$ it is

$$
\left|\mathcal{E}_{3}^{*}\right|=o\left(\left|V^{*}\right|\right) .
$$

Proof. By (9), (15), (16) and using $T=n^{1+\alpha}$ with $\alpha>0$ a constant we have

$$
\begin{aligned}
& \left|\mathcal{E}_{3}^{*}\right|=o\left(\left|V^{*}\right|\right) \Longleftrightarrow T^{2+3 \varepsilon} \cdot \log T / A^{2}=o\left(T^{4 / 3+\varepsilon} / A^{2 / 3}\right) \Longleftrightarrow \\
\Longleftrightarrow & T^{2 / 3+2 \varepsilon} \cdot \log T / A^{4 / 3}=o(1) \Longleftrightarrow \frac{n^{2} \cdot \log T}{T^{2-2 \varepsilon} \cdot(\log n)^{2 / 3}}=o(1) \Longleftrightarrow \\
\Longleftrightarrow & n^{2-(1+\alpha)(2-2 \varepsilon)} \cdot(\log n)^{1 / 3}=o(1) \Longleftarrow(1+\alpha) \cdot(2-2 \varepsilon)>2 \Longleftrightarrow \\
\Longleftrightarrow & \varepsilon<\alpha /(1+\alpha) .
\end{aligned}
$$

Lemma 9. For $0<\varepsilon<1 /(5 \cdot(1+\alpha))-1 / 10$ it is

$$
s_{2,2}\left(\mathcal{G}^{*} ; \mathcal{E}_{4}^{*}\right)=o\left(\left|V^{*}\right|\right) .
$$

Proof. By (9), (15), (18) and using $T=n^{1+\alpha}$ with $\alpha>0$ a constant we infer

$$
\begin{aligned}
& s_{2,2}\left(\mathcal{G}^{*} ; \mathcal{E}_{4}^{*}\right)=o\left(\left|V^{*}\right|\right) \Longleftrightarrow T^{1 / 2+6 \varepsilon}=o\left(T^{4 / 3+\varepsilon} / A^{2 / 3}\right) \Longleftrightarrow \\
\Longleftrightarrow & A^{2 / 3} / T^{5 / 6-5 \varepsilon}=o(1) \Longleftrightarrow n^{(1+\alpha)(1 / 2+5 \varepsilon)-1} \cdot(\log n)^{1 / 3}=o(1) \Longleftarrow \\
\Longleftarrow & (1+\alpha) \cdot(1 / 2+5 \cdot \varepsilon)<1 \Longleftrightarrow \varepsilon<1 /(5 \cdot(1+\alpha))-1 / 10 .
\end{aligned}
$$


Lemma 10. For $0<\varepsilon<1 /(8 \cdot(1+\alpha))$ it is

$$
s_{2,3}\left(\mathcal{G}^{*} ; \mathcal{E}_{4}^{*}\right)=o\left(\left|V^{*}\right|\right) .
$$

Proof. By (9), (15), (19) and using $T=n^{1+\alpha}$ with $\alpha>0$ a constant we have

$$
\begin{aligned}
& s_{2,3}\left(\mathcal{G}^{*} ; \mathcal{E}_{4}^{*}\right)=o\left(\left|V^{*}\right|\right) \Longleftrightarrow T^{2 / 3+5 \varepsilon} \cdot \log T / A^{1 / 3}=o\left(T^{4 / 3+\varepsilon} / A^{2 / 3}\right) \Longleftrightarrow \\
\Longleftrightarrow & A^{1 / 3} \cdot \log T / T^{2 / 3-4 \varepsilon}=o(1) \Longleftrightarrow n^{(1+\alpha) 4 \varepsilon-1 / 2} \cdot(\log n)^{7 / 6}=o(1) \Longleftarrow \\
\Longleftarrow & (1+\alpha) \cdot 4 \cdot \varepsilon<1 / 2 \Longleftrightarrow \varepsilon<1 /(8 \cdot(1+\alpha)) .
\end{aligned}
$$

To satisfy $p=T^{\varepsilon} / t_{0} \leq 1$, we need $T^{\varepsilon} /\left(\left(4 \cdot c_{4}\right)^{1 / 3} \cdot A^{2 / 3} \cdot T^{2 / 3}\right) \leq 1$, which holds by (9) for $0<\varepsilon \leq 2-1 /(1+\alpha)$. For $\varepsilon:=1 /(12 \cdot(1+\alpha))$ and $1 / 12<\alpha<1 / 6$ all assumptions in Lemmas 8, 9, 10 are fulfilled. We delete in $\mathcal{G}^{*}=\left(V^{*}, \mathcal{E}_{3}^{*} \cup \mathcal{E}_{4}^{*}\right)$ one vertex from each 3-element edge $E \in \mathcal{E}_{3}^{*}$, and from each $(2,2)$ - and $(2,3)$ cycle in $\mathcal{G}^{*}$ arising from $\mathcal{E}_{4}^{*}$, and we obtain a subset $V^{* *} \subseteq V^{*}$ with $\left|V^{* *}\right|=$ $(1-o(1)) \cdot\left|V^{*}\right|$. The on the vertex set $V^{* *}$ induced subhypergraph $\mathcal{G}^{* *}$ of $\mathcal{G}^{*}$ contains no 3 -element edges or $(2,2)$ - or $(2,3)$-cycles, i.e. $\mathcal{G}^{* *}=\left(V^{* *}, \mathcal{E}_{4}^{* *}\right)$ with $\mathcal{E}_{4}^{* *}=\mathcal{E}_{4}^{*} \cap\left[V^{* *}\right]^{4}$ is a linear and 4-uniform hypergraph. By (15) and (17) we have

$$
\begin{aligned}
\left|V^{* *}\right| & \geq\left(c_{1}^{\prime} / 2-o(1)\right) \cdot T^{4 / 3+\varepsilon} / A^{2 / 3} \\
\left|\mathcal{E}_{4}^{* *}\right| & \leq 5 \cdot c_{4}^{\prime} \cdot T^{4 / 3+4 \varepsilon} / A^{2 / 3}
\end{aligned}
$$

hence the average degree $t^{3}$ of the subhypergraph $\mathcal{G}^{* *}=\left(V^{* *}, \mathcal{E}_{4}^{* *}\right)$ satisfies

$$
t^{3}=\frac{\left|\mathcal{E}_{4}^{* *}\right|}{\left|V^{* *}\right|} \leq \frac{20 \cdot c_{4}^{\prime} \cdot T^{4 / 3+4 \varepsilon} / A^{2 / 3}}{\left(c_{1}^{\prime} / 2-o(1)\right) \cdot T^{4 / 3+\varepsilon} / A^{2 / 3}}=\frac{40 \cdot c_{4}^{\prime}}{c_{1}^{\prime}-o(1)} \cdot T^{3 \varepsilon}:=t_{1}^{3} .
$$

Since $\mathcal{G}^{* *}$ is linear we can now apply Theorem 2 and, using (9), we find in time

$$
O\left(\left|\mathcal{E}_{4}^{* *}\right|+\frac{\left|V^{* *}\right|^{3}}{t_{1}^{3-\delta}}\right)=O\left(\frac{T^{4 / 3+4 \varepsilon}}{A^{2 / 3}}+\frac{T^{4+\varepsilon \delta}}{A^{2}}\right)=O\left(\frac{n^{3+\delta / 12}}{\log n}\right)
$$

for any $\delta>0$, an independent set $I$ of size

$$
\begin{aligned}
|I| & =\Omega\left(\frac{\left|V^{* *}\right|}{t} \cdot(\log t)^{\frac{1}{3}}\right)=\Omega\left(\frac{\left|V^{* *}\right|}{t_{1}} \cdot\left(\log t_{1}\right)^{\frac{1}{3}}\right)= \\
& =\Omega\left(\frac{T^{4 / 3+\varepsilon} / A^{2 / 3}}{T^{\varepsilon}} \cdot\left(\log T^{\varepsilon}\right)^{\frac{1}{3}}\right)=\Omega\left(\frac{T^{4 / 3}}{A^{2 / 3}} \cdot(\log T)^{\frac{1}{3}}\right)= \\
& =\Omega\left(\frac{n}{(\log n)^{\frac{1}{3}}} \cdot(\log T)^{\frac{1}{3}}\right)=\Omega(n) \quad \text { since } T=n^{1+\alpha} \text { and } \alpha>0 \text { is constant. }
\end{aligned}
$$

By choosing a sufficiently small constant $c>0$ in (9), we obtain in $\mathcal{G}^{* *}$ and hence in $\mathcal{G}$ an independent set of size $n$, which yields a desired set of $n$ grid-points in the $T \times T$-grid such that the area of the convex hull of every four distinct of these $n$ points is at least $A=\Theta\left(T^{2} \cdot(\log n)^{1 / 2} / n^{3 / 2}\right)$. After rescaling we have $n$ points in 
$[0,1]^{2}$ such that the area of the convex hull of every four distinct of these $n$ points is at least $\Omega\left((\log n)^{1 / 2} / n^{3 / 2}\right)$. In comparing the running times $(20)$ and $(24)$ we get the overall time bound $O\left(T^{25 / 2} \cdot(\log n)^{2} / n^{6}\right)$ for $0<\delta<1$. For $\alpha=1 / 11$, say, we have the time bound $O\left(n^{84 / 11} \cdot(\log n)^{2}\right)=O\left(n^{8}\right)$. Indeed, we achieve the time bound $O\left(n^{13 / 2+\delta}\right)$ for any $\delta>0$ by choosing $\varepsilon:=1 /(C \cdot(1+\alpha))$ and $\alpha:=1 /(C-1)$, where $C \geq 12$ is a large enough constant, i.e. $C>1+25 /(2 \cdot \delta)$.

\section{Final Remarks}

It seems that our approach does also work for improving algorithmically the lower bound on $\Delta_{k}(n)$ from [5] for $k=5$ by a poly-logarithmic factor, but not for arbitrary values $k \geq 6$. However, this is work in progress. To decide, whether our algorithm yields optimal solutions, one needs an upper bound. However, at present only $\Delta_{k}(n)=O(1 / n)$ for fixed integers $k \geq 4$ is known, see Schmidt [19].

\section{References}

1. M. Ajtai, J. Komlós, J. Pintz, J. Spencer and E. Szemerédi, Extremal Uncrowded Hypergraphs, Journal of Combinatorial Theory Ser. A, 32, 1982, 321-335.

2. G. Barequet, A Lower Bound for Heilbronn's Triangle Problem in d Dimensions, SIAM Journal on Discrete Mathematics 14, 2001, 230-236.

3. G. Barequet, The On-Line Heilbronn's Triangle Problem in Three and Four Dimensions, Proceedings COCOON'02', LNCS 2387, Springer, 2002, 360-369.

4. C. Bertram-Kretzberg and H. Lefmann, The Algorithmic Aspects of Uncrowded Hypergraphs, SIAM Journal on Computing 29, 1999, 201-230.

5. C. Bertram-Kretzberg, T. Hofmeister and H. Lefmann, An Algorithm for Heilbronn's Problem, SIAM Journal on Computing 30, 2000, 383-390.

6. P. Brass, An Upper Bound for the d-Dimensional Heilbronn Triangle Problem, preprint, 2003.

7. R. A. Duke, H. Lefmann and V. Rödl, On Uncrowded Hypergraphs, Random Structures \& Algorithms 6, 1995, 209-212.

8. A. Fundia, Derandomizing Chebychev's Inequality to find Independent Sets in Uncrowded Hypergraphs, Random Structures \& Algorithms, 8, 1996, 131-147.

9. T. Jiang, M. Li and P. Vitany, The Average Case Area of Heibronn-type Triangles, Random Structures \& Algorithms 20, 2002, 206-219.

10. J. Komlós, J. Pintz and E. Szemerédi, On Heilbronn's Triangle Problem, Journal of the London Mathematical Society, 24, 1981, 385-396.

11. J. Komlós, J. Pintz and E. Szemerédi, A Lower Bound for Heilbronn's Problem, Journal of the London Mathematical Society, 25, 1982, 13-24.

12. H. Lefmann, On Heilbronn's Problem in Higher Dimension, Combinatorica 23, 2003, 669-680.

13. H. Lefmann and N. Schmitt, A Deterministic Polynomial Time Algorithm for Heilbronn's Problem in Three Dimensions, SIAM Journal on Computing 31, 2002, 1926-1947.

14. K. F. Roth, On a Problem of Heilbronn, Journal of the London Mathematical Society 26, 1951, 198-204. 
15. K. F. Roth, On a Problem of Heilbronn, II, Proc. of the London Mathematical Society (3), 25, 1972, 193-212.

16. K. F. Roth, On a Problem of Heilbronn, III, Proc. of the London Mathematical Society (3), 25, 1972, 543-549.

17. K. F. Roth, Estimation of the Area of the Smallest Triangle Obtained by Selecting Three out of $n$ Points in a Disc of Unit Area, Proc. of Symposia in Pure Mathematics, 24, 1973, AMS, Providence, 251-262.

18. K. F. Roth, Developments in Heilbronn's Triangle Problem, Advances in Mathematics, 22, 1976, 364-385.

19. W. M. Schmidt, On a Problem of Heilbronn, Journal of the London Mathematical Society (2), 4, 1972, 545-550. 